\title{
Scenarios for the spread of COVID-19 analyzed by the TVBG-SEIR spline model
}

\author{
Ognyan Kounchev ${ }^{1}$, Georgi Simeonov ${ }^{1}$, Zhana Kuncheva ${ }^{2}$ \\ ${ }^{1}$ Institute of Mathematics and Informatics \\ Bulgarian Academy of Sciences \\ Sofia, Bulgaria \\ kounchev@math.bas.bg, gsimeonov@math.bas.bg \\ ${ }^{2}$ C4X Discovery Ltd \\ London, UK \\ z.kuncheva@gmail.com
}

Received: 16 September 2020, accepted: 8 March 2021, published: 15 March 2021

\begin{abstract}
We develop a novel TVBG-SEIR spline model for analysis of the coronavirus infection (COVID-19). It aims to analyze the long-term global evolution of the epidemics "controlled" by the introduction of lockdown/open up measures by the authorities. The incorporation of different "lockdown scenarios" varying in time permits to analyze not only the primary epidemic wave but also the arising secondary wave and any further waves.
\end{abstract}

The model is supplied by a web-based Scenario Building Tool for COVID-19 (called shortly SBTCOVID19) which may be used as a decision support software by (health) policy makers to explore various scenarios. This can be achieved by controlling/changing the scale of the containment measures (home and social isolation/quarantine, travel restrictions and other) and to assess their effectiveness. In particular, the SBT-COVID19 Tool permits to assess how long the lockdown measures should be maintained.
Keywords-COVID-19; SEIR model; spline models; time-varying transmission and removed rates; timevarying basic reproduction number

\section{INTRODUCTION}

\section{A. Context}

The coronavirus infection (COVID-19) started in December 2019 in Wuhan, China, and has quickly spread out to almost all other countries in the world. The possible catastrophic impact on the economy and health system has required the active intervention of the authorities to introduce various containment measures. This caused the necessity for creating an appropriate model to plan the type, scale and duration of the measures.

Mathematical models are traditionally used to analyze the long-term global evolution of epidemics, to determine the potential and severity

Copyright: (C) 2021 Kounchev et al. This article is distributed under the terms of the Creative Commons Attribution License (CC BY 4.0), which permits unrestricted use, distribution, and reproduction in any medium, provided the original author and source are credited.

Citation: Ognyan Kounchev, Georgi Simeonov, Zhana Kuncheva, Scenarios for the spread of

COVID-19 analyzed by the TVBG-SEIR spline model, Biomath 10 (2021), 2103087,

http://dx.doi.org/10.11145/j.biomath.2021.03.087 
of an outbreak, and to provide critical information for identifying the type of disease interventions and intensity. One of the widely used mathematical models of long-term spreading of epidemics are the so-called deterministic compartmental models (SIR/SEIR type models [4]) which reflect adequately the clinical progression of the virus spreading, epidemiological status of the individuals, and the intervention measures. One of the main purposes of applying such models is to assess how the expensive containment measures imposed by the authorities (home and social isolation/quarantine, travel restrictions, etc.) can effectively reduce the basic reproduction number, transmission rate and risk of the disease. In particular, it is essential to assess how the expensive, resource-intensive measures implemented by the authorities can contribute to the prevention and control of the COVID-19 infection, and how long they should be maintained.

However the classical SIR/SEIR models have been primarily studied in the case where the main parameters - the Transmission rate $\beta$ (reflecting the virus spread by infected individuals) and the Removed rate $\gamma$ (reflecting the hospitalization/isolation measures) - remain constant during the whole period of interest. This does not reflect in a proper way the extremely dynamic behavior of such measures during the COVID-19 and similar epidemics, resulting from the imposition of intensive containment measures by the authorities.

\section{B. Aims and Methods Summary}

It is important to extend the classical SIR/SEIR models by creating new models for the dynamics of the transmission rates $\beta(t)$ (sometimes referred to as Beta) and removed rates $\gamma(t)$ (sometimes referred to as Gamma). The main aim of the present research is to introduce a novel splinebased SEIR model with time-varying $\beta(t), \gamma(t)$ parameters, or abbreviated TVBG-SEIR model, which is used to estimate the practical implications of the public health interventions and containment measures. We have designed a Scenario Building Tool for COVID-19 (SBT-COVID19 Tool) based on the TVBG-SEIR model, which may be used as a Decision Support Tool to assist the health decision- and policy-makers in creating predictive scenarios. It may be used to assess the impact of previous public health interventions, and to plan quantitatively and qualitatively the introduction of future containment measures for achieving the necessary objectives.

For formulating our model, we use deterministic spline Ansatz: the transmission rates $\beta(t)$ and the removal rates $\gamma(t)$ are modeled by splines with two nodes - Node1, Node2 (the same nodes for both $\beta(t)$ and $\gamma(t)$ ) - within the time interval of interest - from StartDate until Today. This Ansatz allows to properly model the dynamics due to the introduction of containment measures by the authorities in two steps. The purpose of fitting of the TVBG-SEIR model is to identify the nodes of the splines and the three values of $\beta(t)$ and $\gamma(t)$ on Node 1, Node 2 , and Today date. It is assumed that $\beta(t)$ and $\gamma(t)$ are constant in the time interval [StartDate, Node1], and of $\beta(t)$ is monotone decreasing while $\gamma(t)$ is monotone increasing function. The TVBG-SEIR model with time-varying $\beta(t)$ and $\gamma(t)$ will be fitted simultaneously to two sets of data: the daily infected cases (or their cumulative vector), and the removed cases (which are all removed cases until a certain date).

The choice of just two nodes of the splines for the rates $\beta(t)$ and $\gamma(t)$ seems to be appropriate for models of historical data (until Today) for not very long periods of time. These models are used as a basis for creation of "prediction scenarios" starting from Today, with a prediction perspective of about 2 months horizon. These models are updated every day (by the arrival of the official daily data), and the scenarios are renewed accordingly. In a more mathematical language "scenario" means choice of control parameters $\beta(t)$ and $\gamma(t)$ in the form of splines defined after Today, which determine the SEIR model to be defined below. One may choose these scenarios in infinitely many ways. The main objective of our approach is to choose such scenarios $\beta(t)$ and $\gamma(t)$ for which the SEIR model generates curves which satisfy some reasonable restrictions, e.g. the number of infected 
O. Kounchev, G. Simeonov, Zh. Kuncheva, Scenarios for the spread of COVID-19 analyzed by ...

daily cases does not explode too abruptly. Let us emphasize that these are just "possible prediction scenarios" but not extrapolations in the classical sense of the word.

The web-based SBT-COVID19 Tool was designed for visualization of the results of the fitted model (the daily infected cases), and for creating prediction scenarios for the daily infected cases during the next two month horizon, by controlling the future values of Beta and Gamma. It is described in detail in Section VII, and is available at the links

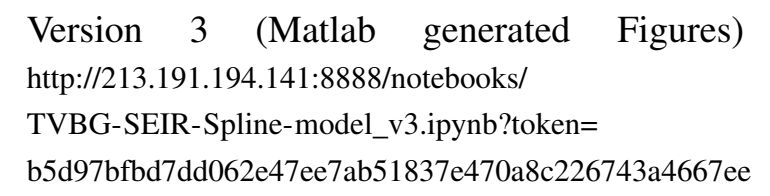

Version 4 (Python generated Figures)
http://213.191.194.141:8889/notebooks/

TVBG-SEIR-Spline-model_v4.ipynb?token=

b5d97bfbd7dd062e47ee7ab51837e470a8c226743a4667ee

\section{Objectives}

In the case of the usual seasonal flu the main parameters of the spread of the viruses are the transmission rate $\beta$ which reflects the power of the transmission of the virus from infected people to susceptibles, the recovery rate $\gamma$ which is reciprocal to the recovery period (which is the sum of "recovery to health cases" + "isolated sick cases" + "mortality due to the sickness cases"), and the parameter $\sigma$ which is the reciprocal to the incubation period. Due to the long incubation period and large number of asymptomatic or mildsymptomatic cases, COVID-19 has proved to be very insidious and requires intensive emergency measures from the authorities to reduce the transmission rate $\beta$ and to increase the recovery rate $\gamma$. For comparison, in the case of the seasonal flu no intensive containment measures are necessary to be undertaken by the authorities.

For containing COVID-19, the authorities have introduced very strong measures which have essentially influenced the dynamics of the parameters $\beta$ and $\gamma$. For the majority of states these measures have been introduced not only in one step but most often in two steps. It depends on every society how fast these measures will be implemented in life. There are two types of measures: for example, closing schools, pubs, restaurants, traveling national or international routes, social meetings, wearing masks, reduce directly the transmission rate of the disease $\beta$ (further we will call these measures shortly Beta measures); on the other hand the fast identification and medication of virus spreaders, hospitalization, quarantining and similar, increase the rate $\gamma$ of "removal from the group of virus spreaders" (further we will call these shortly Gamma measures). It is important to assess how these expensive and resource intensive measures implemented by the authorities can contribute to the prevention and control of the COVID-19 infection, and how long they should be maintained, [12], [13]. In order to meet the challenge of Controlled spread of the COVID-19 (and similar) epidemics, one needs to develop new mathematical models which better describe reality. Based on the widely used conventional epidemiological model SEIR, in the present research we propose a new model TVBGSEIR which incorporates a specific spline model for the time-varying transmission $\beta$ and removal $\gamma$ rates.

\section{Data Acknowledgement}

We acknowledge the following data sources used in the present research of COVID-19:

- HDX Humanitarian Data Exchange V1.39.3 time_series_covid19_confirmed_global.csv time_series_covid19_deaths_global.csv time_series_covid19_recovered_global.csv https://data.humdata.org/dataset/ novel-coronavirus-2019-ncov-cases

- Kaggle SRK Sudalairaj Kumar https://www.kaggle.com/sudalairajkumar/ novel-corona-virus-2019-dataset\# covid 19 data.csv

The paper is organized as follows: In Section I we recall the deterministic SEIR model and introduce some notions and notations. In Section II 
we introduce the discretization of the SEIR model which is used in the algorithms. In Section III we introduce and provide all technical details of the TVBG-SEIR spline model. In Section IV we provide an application of the TVBG-SEIR model to Bulgarian data, which are used to illustrate the work of the SBT-COVID19 Tool for prediction scenarios. In Section V and Section VI we provide more examples of analysis, for the Italian data, and for the German data. In Section VII we describe the technical details of the SBT-COVID19 Tool for prediction scenarios. In Section VIII we provide some recent references about models with timevarying transmission rates and their fitting to the data (calibration).

\section{THE CLASSICAL DETERMINISTIC SEIR MODEL: NOTIONS AND NOTATIONS}

The main purpose of the authors' presentation is to provide all notations and formulas as fully as possible, to enable the replicability of the calculations and experiments in the paper. Thus we also provide a detailed account of the discretization of the well-known classical SEIR model.

\section{A. Compartmental models}

Compartmental models are a framework used to model in an adequate way the dynamics of infectious disease. The population is divided into compartments, with the assumption that every individual in the same compartment has the same characteristics. This framework has been developed for the first time in the paper of Kermack and McKendrick in 1927 [2]. One may use a deterministic approach using a system of ODEs or a stochastic approach which is more complicated. The deterministic approach is what we follow, namely our choice is the SEIR model. The main reason for choosing SEIR model against its simpler relative SIR model, is the long incubation period of COVID-19, hence, the large "exposed cases" compartment to be defined below.

For a detailed and excellent introduction to the compartmental models we refer to the monograph
[4]. We provide a short description of the deterministic SEIR model which will be the main approach in our research.

The classical SEIR model is based on the consideration of four compartments, $C_{S}, C_{E}, C_{I}$ and $C_{R}$ which are described as follows: Compartment $C_{S}$ : its size is $S r(t)$ - the number of "susceptible" people at time t. Usually, at the start, $S(0)=N$ is the whole population of the country under consideration. It is supposed that nobody has automatic immunity against the virus, i.e. everybody is susceptible.

Compartment $C_{E}$ : its size is $\operatorname{Er}(t)-$ the number of "exposed" people at time $t$ - these are the people who are "virus carriers" but are not "virus spreaders"; the virus is in a latent form, and usually they do not show symptoms of sickness. For different viruses the incubation (latent) period is very different - for the coronavirus it was recently statistically estimated that the average incubation period is 11.5 days [3]. Not everybody in $C_{E}$ may become "virus spreader", i.e. move to the next compartment $C_{I}$. Practically, the compartment $C_{E}$ does not enter the official statistics since it is not observable, but it is very important for the modeling of the dynamics of the virus spread. This compartment is missing in the simpler SIR model. Compartment $C_{I}$ : its size is $\operatorname{Ir}(t)$ - the number of infectious cases at time $\mathrm{t}$ - these are the people who are "virus spreaders", majority of them show some symptoms, although they may not show any symptoms (asymptomatic). It is important to understand in the modeling that many people who are diagnosed positively are almost immediately hospitalized or quarantined, hence they go to compartment $C_{R}$, but they have stayed in $C_{I}$ only until they have been diagnosed (and these are the official data which we obtain - denoted further by Idata(t) ). Compartment $C_{R}$ : its size is $\operatorname{Rr}(t)$ - the number of recovered or deceased (or immune) individuals, which are all called "removed". Normally they come from compartment $C_{I}$ after becoming healthy and no more virus spreaders. Officially these data are provided in a cumulative way. 
O. Kounchev, G. Simeonov, Zh. Kuncheva, Scenarios for the spread of COVID-19 analyzed by ...

\section{B. Official data available for modeling purposes}

We do not have the "reality data" $S r(t), \operatorname{Er}(t)$, $\operatorname{Ir}(t), \operatorname{Rr}(t)$. The available official data $\operatorname{Idata}(t)$ are the daily "new infected cases" with COVID19 , and these are normally people with serious symptoms. These are the cases which have been tested and registered officially at the hospitals. The majority of them are almost immediately hospitalized or quarantined, hence, they are almost immediately moved from compartment $C_{I}$ to compartment $C_{R}$. However it is well known that for seasonal flu (and it is considered to be similar for COVID-19) the size of $C_{I}$ is much bigger than that indicated by the official data $\operatorname{Idata}(t)$, and we have the inequality $\operatorname{Idata}(t) \leq \operatorname{Ir}(t)$.

The officially announced data $\operatorname{Rdata}(t)$ contains the cumulative number of recovered cases and the $\operatorname{Ddata}(t)$ is the cumulative number of fatalities.

A main point of the modeling paradigm for COVID-19 (and similar virus infections) is that, for a certain segment of the society (in this case, the younger people), the infection symptoms do not differ essentially from a seasonal flu, hence the number of unreported cases (those which are in compartment $C_{I}$ but not in $\operatorname{Idata}(t)$ for every time $t$ ) may be much bigger, thus in the above inequalities more appropriate is to use the symbol "«", which denotes roughly speaking "much less". In the case of the seasonal flu it may be even 100 times less.

\section{Definition of the continuous SEIR model}

The main point of developing the compartmental deterministic SEIR model is to provide some tractable approximations $\mathrm{S}(\mathrm{t}), \mathrm{E}(\mathrm{t}), \mathrm{I}(\mathrm{t}), \mathrm{R}(\mathrm{t})$ to the above time series of the "reality data" $\operatorname{Sr}(\mathrm{t}), \operatorname{Er}(\mathrm{t}), \operatorname{Ir}(\mathrm{t}), \operatorname{Rr}(\mathrm{t})$. The most widely used is the model based on a system of Ordinary Differential Equations with variables $S(t), E(t), I(t), R(t)$ which is given as follows:

$$
\begin{aligned}
S^{\prime}(t) & =-\beta(t) S(t) I(t) / N \\
E^{\prime}(t) & =\beta(t) S(t) I(t) / N-\sigma E(t) \\
I^{\prime}(t) & =\sigma E(t)-\gamma(t) I(t) \\
R^{\prime}(t) & =\gamma(t) I(t)
\end{aligned}
$$

Let us explain the notations and the correspondence to the "reality data" of the Compartmental model:

Here the term $\beta(t) I(t) / N$ expresses the rate at which new individuals (as a proportion of the total population size) are infected by the already infectious I(t) individuals, (cf. Keeling-Rohani, (2008), p.18). Here and further $\beta(t)$ is called Transmission rate of the infection, which we call further simply Beta.

As already said, the coefficient $\gamma(t)$ is the Removal rate; it is determined by the reciprocal of the infectious period, after which either the person is recovered (and no more infectious) or dead (again, no more infectious). Here and further $\gamma(t)$ is called Removal rate, and sometimes we call it simply Gamma. The coefficient $\sigma$ is the latent rate, or the rate of "becoming symptomatic" (where $1 / \sigma$ is the average of the incubation period). In the present paper we use the constant value $\sigma=1 / 5.2$ which represents a reasonable approximation, as the recent research shows, see [3].

The variable $S(t)$ corresponds to the reality data $\operatorname{Sr}(t)$. The quantity $\sigma E(t)$ is equal to the daily new infectious cases $\operatorname{Idata}(t)$. However, the quantity $I(t)$ of the SEIR model is equal to the so-called Active Cases which are defined by the equation

$$
A C(t)=\text { Total_Infected }(t)-R r(t)
$$

Here Total_Infected $(t)$ is the cumulative sum of $\operatorname{Idata}(t)$ until the date $t$.

The usual applications of the SEIR model are with constant rates $\beta(t)$ and $\gamma(t)$. One assumes that the initial values $S(0), E(0), I(0)$ and $R(0)$ are given and the system is solved for the times $t \geq 0$, where $\mathrm{t}$ is an integer. It is assumed that the following equation holds

$$
N=S(0)+E(0)+I(0)+R(0),
$$


O. Kounchev, G. Simeonov, Zh. Kuncheva, Scenarios for the spread of COVID-19 analyzed by ...

where $N$ is the total population in the country $\mathrm{XX}$. Obviously, since the sum

$$
S^{\prime}(t)+E^{\prime}(t)+I^{\prime}(t)+R^{\prime}(t)=0
$$

it follows that equation (2) holds for every time $t \geq 0$.

\section{DisCRETIZATION OF THE SEIR MODEL}

In practice one uses a discretization of the continuous SEIR model. In the current work we use the following discretization of the SEIR model which is derived from the Euler method for approximate solution of the initial value problem (1):

$$
\begin{aligned}
S_{(n+1)} & =S_{n}-\left(\beta_{n} S_{n} I_{n}\right) / N \\
\left.E_{(} n+1\right) & =E_{n}+\left(\beta_{n} S_{n} I_{n}\right) / N-\sigma E_{n} \\
\left.I_{(} n+1\right) & =I_{n}+\sigma E_{n}-\gamma_{n} I_{n} \\
R_{(n+1)} & =R_{n}+\gamma_{n} I_{n}
\end{aligned}
$$

Here $S_{n}, E_{n}, I_{n}$ and $R_{n}$ are respectively the values of $S(t), E(t), I(t)$ and $R(t)$ on the day $t=n$, and the initial values for day $n=0$ are $S_{0}, E_{0}$, $I_{0}$ and $R_{0}$. The above system is iteratively solved for integers $n \geq 0$. We assume that the size $N$ of the population remains unchanged (hence no usual birth and mortality are taken into account). As in the continuous case, the total sum of the above is assumed to satisfy

$$
N=S_{n}+E_{n}+I_{n}+R_{n}
$$

which makes one of the equations in (3) redundant. It is well known that the above Euler method for approximating the solution of (1) is less accurate than the Runge-Kutta which is widely used, see e.g. [11]. Again, it is very important for the modeling process to realize what is the correspondence between the variables of the discrete model and the officially announced data. On the day $n$ the value $R_{n}$ corresponds to the sum of the cumulative recovered plus fatalities data, i.e. to $\operatorname{Rdata}(n)+\operatorname{Death}(n)$. The announced daily data of newly infected $\operatorname{Idata}(n)$ correspond to the amount $\sigma E_{n}$ which is clear from the third equation in (3). Below we use this correspondence to define the quadratic function $F(\theta)$ for fitting our models.
Remark: One has to note that the continuous model (1) and the above discrete approximation (3) have essential differences in the long-term behavior which has been the subject of much research. It is important to note that the qualitative properties of the solution to the differential equation and of the discrete equation differ essentially - the continuous case is simpler as usual.

\section{THE TVBG-SEIR MODEL}

The SEIR models have proved to be very efficient in situations where the main parameters $\beta$ and $\gamma$ are constants, in natural conditions, where no special control by the authorities is exercised, i.e. no intervention measures are undertaken to change the transmission and the removal rates in the course of the epidemics. This is very often the case with the seasonal flues, where the medical authorities do not undertake actively special measures to restrict the social behavior of the citizens, although nowadays the vaccinations change the "natural" picture. However due to the specific of the COVID-19 the situation has become more dramatic and it has required the interference of the governments in order to avoid the overloading of the National Health systems. The authorities have introduced very strong restrictive measures which have essentially influenced the dynamics of the parameters $\beta, \gamma$. For the majority of the states these measures have been introduced not only in one step but most often at least in two steps. In view of the above it makes sense to seek for mathematical models which try to model as best as possible the dynamics of the parameters $\beta$ and $\gamma$. We have decided for spline structure with two important breakpoint nodes, Node1, Node 2 - which reflect the control exercised by the authorities in the form of restriction measures. Also, it is natural to assume that between the dates the control measures change the parameters $\beta(t)$ and $\operatorname{gamma}(t)$ in a monotone way, i.e. $\beta(t)$ is decreasing whereas $\gamma(t)$ is increasing.

\section{A. Technical Description of the TVBG-SEIR model}

1) We denote the StartDate by $T_{1}$; this corresponds to a date when the first cases of COVID-19 
are announced, eventually we may choose $T_{1}$ to be a date when the steeper growth of the epidemic starts. We denote by $T_{4}$ the EndDate (usually chosen to be Today).

2) We choose two interior nodes in the interval $\left[T_{1}, T_{4}\right]$ for the interpolation splines modeling the coefficients $\beta(t), \gamma(t):$ Node $1=T_{2}$ and Node $2=T_{3}$. This corresponds to two steps of the introduction of restrictive measures imposed by the authorities of the country XX. Normally, the date $T_{2}$ may be the first restrictive measures date, or a date close to it, and $T_{3}$ may be the Second restrictive measures date, or a date close to it.

3) The model is supposed to reflect the natural expectation that once there are official restrictions, they will implicate an essential change in the Transmission and Removed rates although not immediately. We assume that the rate $\beta(t)$ is monotone decreasing with the time, which corresponds to the natural expectation that the more restrictive the measures the smaller the Transmission rate. Respectively, the rate $\gamma(t)$ is assumed to be monotone increasing, meeting the expectation that the stronger the measures, the bigger the removal rate.

4) We assume that $\beta(t)$ and $\gamma(t)$ are constant between the start date $T_{1}$ and the first node $T_{2}$, i.e. $\beta\left(T_{1}\right)=\beta\left(T_{2}\right)$ and $\gamma\left(T_{1}\right)=\gamma\left(T_{2}\right)$. This corresponds to the "still" life of the society (without containment measures) when the rates $\beta(t)$ and $\gamma(t)$ are nearly a constant.

5) To be more precise, the splines which we consider are not the usual polynomial, but the so-called exponential splines depending on a parameter in the exponent, which makes a fast decay to the next target value of the $\beta(t)$ rate; respectively this makes fast increasing to the target value of the rates $\gamma(t)$. This corresponds to the expectation that the speed by which the society switches from one level of the restrictive measures to another is relatively fast, and it is reflected by the size of the exponent we decide to choose. In fact, we use shape preserving exponential splines which are just $C^{1}$ (smooth) and do not need additional boundary conditions. Alternatively, one may use $C^{2}$ (twice differentiable) exponential splines which would be more technical due to the necessity to choose boundary conditions (at the initial and the terminal points). For the practical purposes, there are different spline functions implemented in Matlab/Octave, R, Python.

On the following Figures we see examples of the dynamics of $\beta(t)$ and $\gamma(t)$ rates:

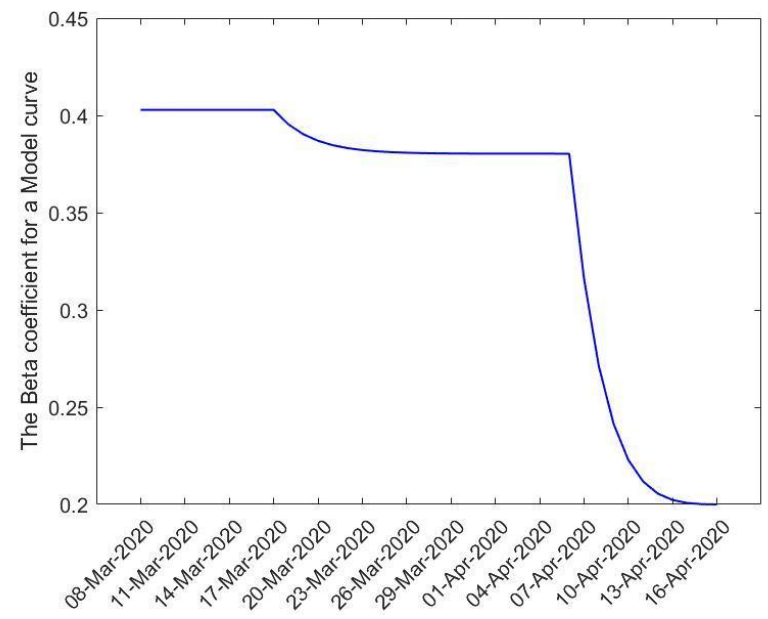

Figure 1. The rates $\beta(t)$ for a Model curve

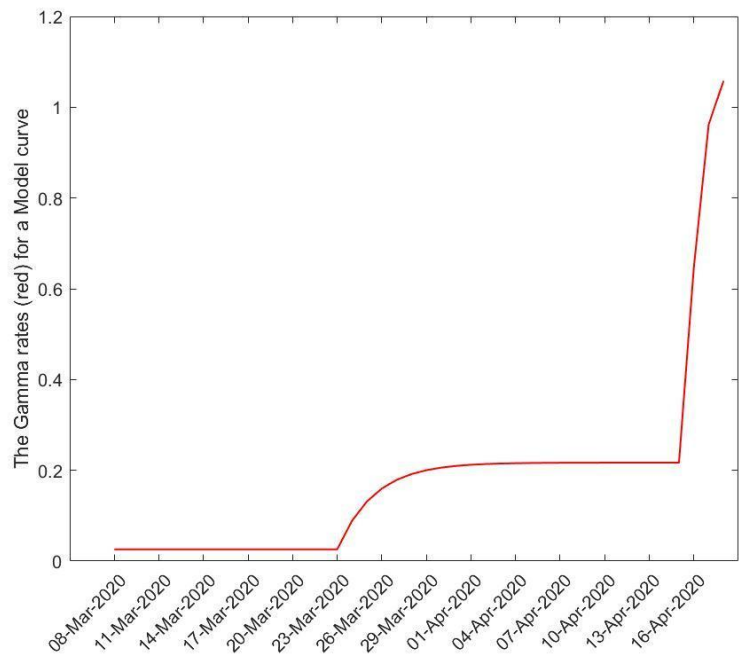

Figure 2. The rates $\gamma(t)$ for a Model curve

6) An important property of the TVBG-SEIR model is that due to the above spline model for the $\beta(t)$ and $\gamma(t)$ parameters, where there is a fast transition to the next target value, a classical SEIR model with constant $\beta(t)$ and $\gamma(t)$ holds during 
larger sub-intervals. In particular, this permits to provide a reliable estimate of the Basic Reproduction Number.

7) The Reproduction number (ratio) is a key variable for all models of epidemics, see [4], [9], [14], [15]. Following [15] (formula (2.4)), for the case of the SEIR models with constant rates $\beta(t)$ and $\gamma(t)$, the Reproduction number is given by the formula

$$
R_{0}=\beta / \gamma,
$$

where we have assumed that the natural birth and mortality rates are small and also equal. Due to the above remark, we may extrapolate the above formula for all time points of interest by putting:

$$
R_{0}=\beta(t) / \gamma(t)
$$

On Figure 3 below we provide the Reproduction Number $R_{0}$, obtained by the last formula, for some specific TVBG-SEIR model:

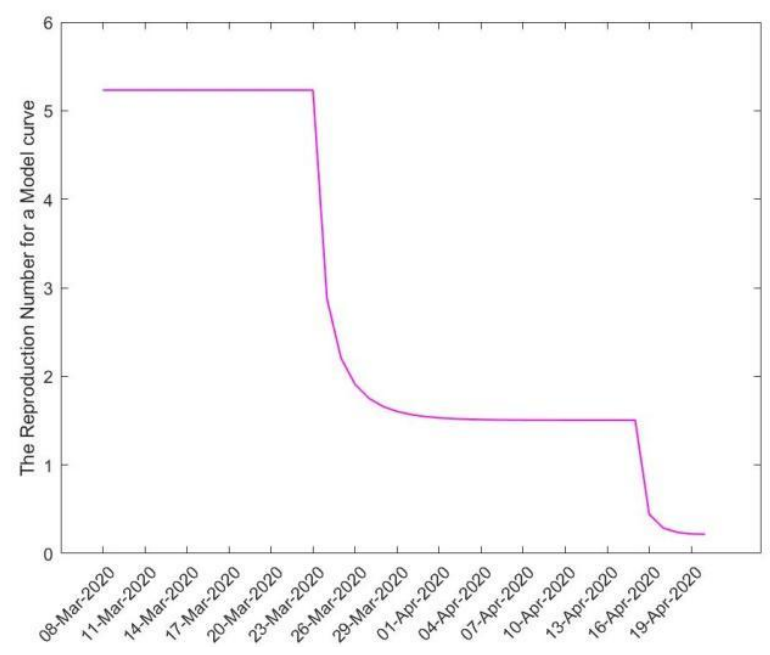

Figure 3.

8) On Figure 1 we see that the nodes of the spline satisfy Node $1=T_{2}=17-$ Mar -2020 and Node $2=T_{3}=7-A p r-2020$. On the other hand on Figure 2 we have chosen a configuration with different nodes, $T_{2}=23-M a r-2020$, and $T_{3}=15-A p r-2020$.

9) In the above examples of the dynamics of $\beta(t)$ and $\gamma(t)$ one sees the exponential factor $\exp \left(0.4 *\left(t-t_{1}\right)\right)$ by which the curve changes from one level at $t=t_{1}$ to the next target level. The coefficient 0.4 is judiciously chosen and may be varied, as well as the exponential function may be replaced by a different proper function.

10) The rates $\beta(t)$ and $\gamma(t)$, are defined as interpolation splines on the subintervals defined by the start date $T_{1}$, the nodes dates $T_{2}, T_{3}$, and the final date $T_{4}$. Thus the whole configuration is defined by eight parameters in total, which we gather in a set $\theta$, given by

$\theta=\left\{T_{2}, T_{3}, \beta\left(T_{2}\right), \beta\left(T_{3}\right), \beta\left(T_{4}\right), \gamma\left(T_{2}\right), \gamma\left(T_{3}\right), \gamma\left(T_{4}\right)\right\}$

11) The data which we use for the fitting of the discrete TVBG-SEIR model are the official data for daily new infected cases $I$ data(t) (or their cumulative vectors $\operatorname{cum}(\operatorname{Idata})(t)$ and the cumulative data for recovered and fatalities.

12) Finally, we fit the Model to the data by optimizing the positions of the two nodes $T_{2}, T_{3}$, and the levels of $\beta(t)$ and $\gamma(t)$, i.e. $F(\theta)$ by varying the parameter set $\theta$ :

$$
\begin{gathered}
F(\theta)=\sum_{j=1}^{n}\left(\operatorname{cum}(\text { Idata })\left(t_{j}\right)-\sigma * \operatorname{cum}(E)\left(t_{j}\right)\right)^{2} \\
\quad+\left(\operatorname{Rdata}\left(t_{j}\right)+\text { Deaths }(t)-R\left(t_{j}\right)\right)^{2} .
\end{gathered}
$$

Here we denoted by $\operatorname{cum}(E)(t)$ the cumulative vector of the solution $E_{n}$ of the discrete SEIR system until the date $t$.

Let us note that there are different possibilities to choose the functional $F(\theta)$ which is used by other authors, and one of the most important arguments is the reliability of the officially announced data.

13) As we said, the minimization of $F(\theta)$ is performed by varying by means of sampling the two nodes $T_{2}<T_{3}$ of the splines in the interval range $\left[T_{1}, T_{4}\right]$; the interpolation values for the splines, $\beta\left(T_{2}\right), \beta\left(T_{3}\right), \beta\left(T_{4}\right), \gamma\left(T_{2}\right), \gamma\left(T_{3}\right), \gamma\left(T_{4}\right)$ are also varied. More details about the possible choice of proper models are provided below in Section V.

14) The "curves" $S_{n}, E_{n}, I_{n}, R_{n}$ of the discrete SEIR model are obtained by solving the system (2) 
O. Kounchev, G. Simeonov, Zh. Kuncheva, Scenarios for the spread of COVID-19 analyzed by ...

with initial conditions given by

$$
S_{1}=N-E_{1}-I_{1}-R_{1},
$$

where $\mathrm{N}$ is the size of the whole population, and also,

$$
\begin{aligned}
\sigma E_{1} & =\operatorname{Idata}(1) \\
R_{1} & =\operatorname{Rdata}(1)+\operatorname{Deaths}(1) \\
I_{1} & =\operatorname{Idata}(1)-R(1) .
\end{aligned}
$$

As defined above, the set $\theta$ contains the parameters which determine the (discrete versions of the) splines for $\beta(t)$ and $\gamma(t)$. Additionally, one may introduce non-negative weights $w_{1}(t)$ and $w_{2}(t)$, which give priority to some of the data.

15) On Figure 4 below we provide the daily data of infected cases $\operatorname{Idata}(t)$ for Bulgaria:

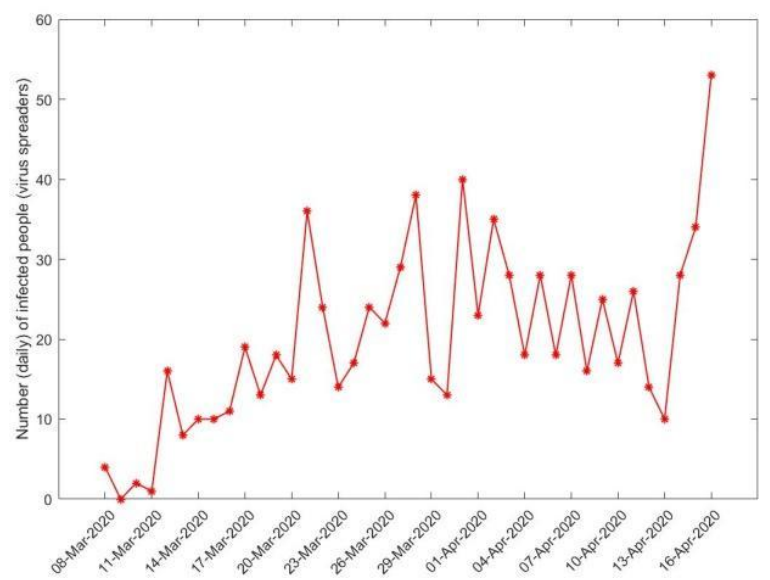

Figure 4.

16) On Figure 5 below the cumulative data for recovered and fatalities are provided. On Figure 6 we provide the fitting of the model curve $\sigma E(t)$ to the above data for Bulgaria, Idata $(t)$.

17) Figure 7 shows the fitting by the model curve $\mathrm{R}(\mathrm{t})$ of the Recovered plus Fatalities data for Bulgaria.

18) It is important to remark that we have applied a "parsimonious" approach for constructing the spline model, by which one has to avoid putting too many nodes in the splines since this will influence the stability of the model, and might cause overfitting, hence would spoil the predictive

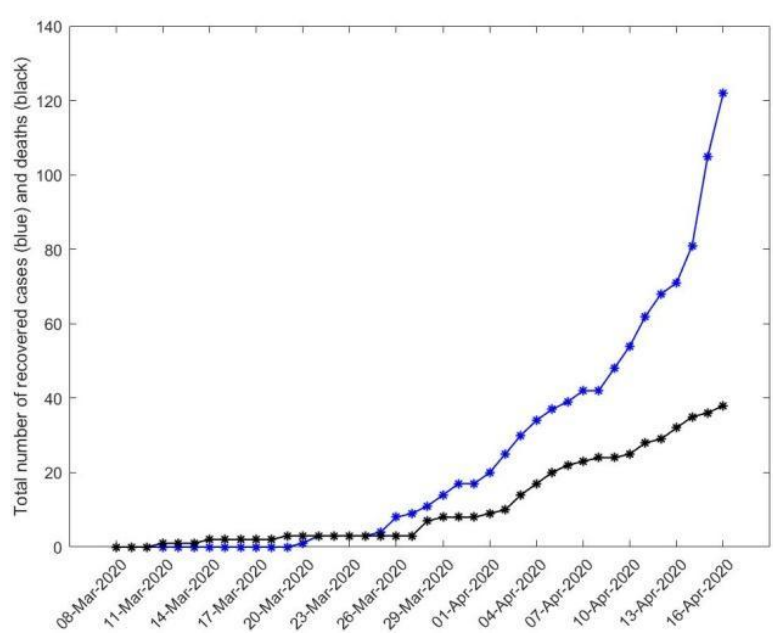

Figure 5.

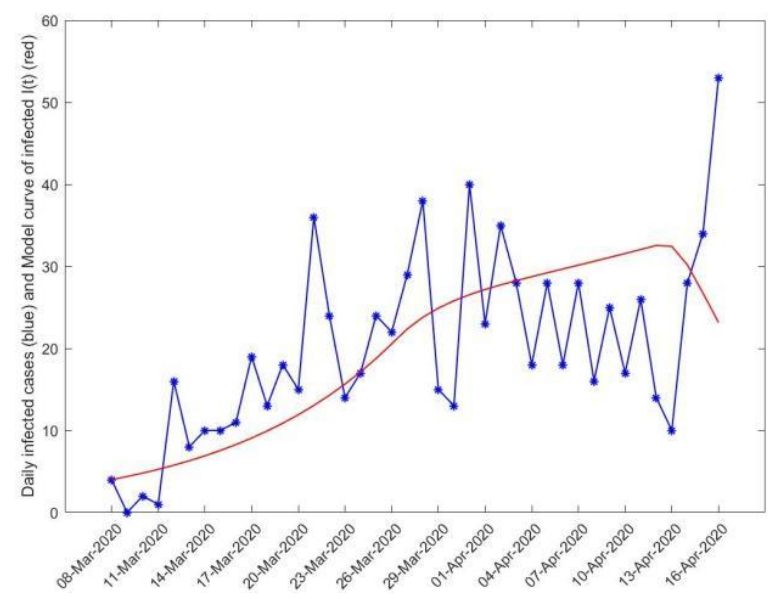

Figure 6.

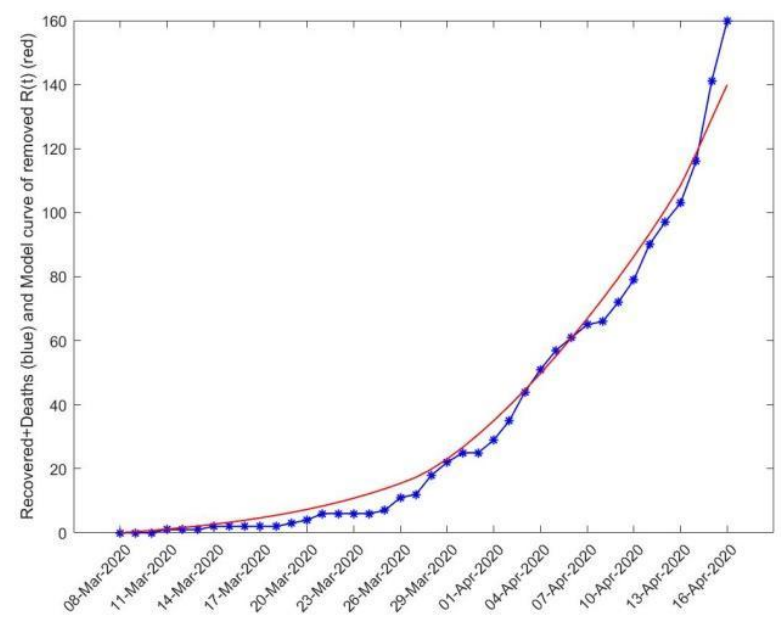

Figure 7. power of the model. 
O. Kounchev, G. Simeonov, Zh. Kuncheva, Scenarios for the spread of COVID-19 analyzed by ...

19) As we said, the result of the minimization of the quadratic functional $F(\theta)$ is a set of parameters $\theta$ for which the minimum is attained, whereby there may be multiple solutions. Once we have found some model based on the parameters $\theta$, we proceed to constructing "prediction scenarios". We choose some date $T_{6}$, which we call Horizon, say at most 2 months from Today $=T_{4}$, by putting $T_{6}=T_{4}+2$ months.

Then a scenario is defined by choosing an additional node $T_{5}$, which is a Third restriction measures date, and the parameters

$$
\left\{\beta\left(T_{5}\right), \beta\left(T_{6}\right), \gamma\left(T_{5}\right), \gamma\left(T_{6}\right)\right\}
$$

We put

$$
\begin{aligned}
& \beta\left(T_{5}\right)=\text { Coef } 1 * \beta\left(T_{4}\right) \\
& \beta\left(T_{6}\right)=\text { Coef } 11 * \beta\left(T_{5}\right) \\
& \gamma\left(T_{5}\right)=\text { Coef } 2 * \gamma\left(T_{4}\right) \\
& \gamma\left(T_{6}\right)=\text { Coef } 22 * \gamma\left(T_{5}\right)
\end{aligned}
$$

The coefficients Coef1, Coef11, Coef2, Coef 22 are used further to control and represent our scenario building in the SBT-COVID19 Tool. Their meaning and choice is explained in detail in Section VIII, where we introduce the Tool.

\section{COVID-19 SPREAD IN BUlgaria IN} OCTOBER-DECEMBER, 2020: APPLICATION TO BULGARIAN COVID-19 DATA AND SCENARIOS GENERATED BY THE SBT-COVID19 TOOL

In the SBT-COVID19 Tool one may find online the results for analyzing of the Covid-19 data and for generating scenarios in the case of Bulgaria, for the period March-August, 2020, (Kounchev et al., (2020), [17]). There we have shown the possibility for a next wave of the infection.

Here we demonstrate how to generate prediction scenarios based on the Bulgarian data for the period 1 October, $2020-3$ January, 2021. In the results provided below, it is clearly visible that the model reflects properly the wave of the epidemic in October-November and its decline at the end of December due to the lockdown imposed on November 25th, 2020. It also hints the appearance of a next wave for certain scenarios, which correspond to special choices of the splines for $\beta(t)$, $\gamma(t)$ after Today date, which model relaxation of the containment measures. We have to emphasize that the date Today and "Third restriction measures date" are the only nodes of the splines for $\beta(t)$, $\gamma(t)$ in the interval after Today date. Hence, the only parameters which determine a "prediction scenario" are the "Third restriction measures date" and the values of $\beta(t), \gamma(t)$ at them.

The SBT-COVID19 Tool will be described in detail in Section VII. We provide the visualizations of the model fitting which are available in the SBTCOVID19 Tool. The thick red curve on the Figures below shows the fitted model curve until Today $=$ $T_{4}$ for the daily new infected cases $\sigma E(t)$ and the blue stars show the official data for them, namely $\operatorname{Idata}(t)$. The thin red curve shows the prediction scenarios, after Today.

Definition. Under scenario we understand a choice of the coefficients Coef 1 , Coef2, which indicate whether we relax the measures (i.e. we set them to $0.2,0.4,0.6,0.8)$, retain the measures $(=1.0)$ or tighten measures (i.e. we set them to $1.2,1.4,1.6,1.8)$ which determine the parameters $\beta(t), \gamma(t)$ of the epidemic after Today date, as well as of the coefficients Coef11, Coef22, which indicate a relaxation (if set equal to $1.2,1.6,1,8$ ) of the two types of measures after the HORIZON date (for which we have three possible choices, namely, 5, 15, 25 days from Today).

1) For Bulgaria we have considered the data from the StartDate which is $T_{1}=1-O c t-2020$, until the end date Today, equal to $T_{4}=3-$ Jan -2021 . Third restrictions date $=T_{5}=$ $28-$ Jan -2021 , and the Horizon date is $T_{6}=$ $22-$ Feb -2021.

2) As we explained in Section IV the minimization of the functional $F(\theta)$ consists of considering many pairs of nodes $T_{2}, T_{3}$ (about 150 for a threemonth period) for the splines $\beta(t), \gamma(t)$. We select the pair $T_{2}, T_{3}$ and the corresponding parameters $\theta$ (which define Model 1 ) for which the minimum $F(\underline{\theta})$ of $F(\theta)$ is attained. However there are also other parameter vectors $\theta$ for which the functional 
$F(\theta)$ attains values very close to the optimal value $F(\underline{\theta})$. We denote these by $\underline{\theta}=\underline{\theta}^{1}, \underline{\theta}^{2}$, $\underline{\theta}^{3}$, etc. These vectors define parameters $\beta^{(j)}, \gamma^{(j)}$, or equivalently, models, which we denote by Model1, Model2, Model3, etc. The curves of the TVBG-SEIR model which correspond to these parameters $\beta^{(j)}, \gamma^{(j)}$ play a very useful role, and serve as an alternative to the Bootstrapping procedure as described in the classical textbooks, see e.g. Hastie and Tibshirani (2009), [16]. Thus, it will provide us also alternative to finding the Confidence intervals for the obtained results.

Let us note that in the example above the maximum value of the functional $F(\theta)$ is 275.90 (taken over all admissible parameters $\theta$ ), while the minimum is 30.96 .

3 ) For the optimistic, Model1, we have found $T_{2}=9-N o v-2020, T_{3}=2-D e c-2020$, with $F(\underline{\theta})=30.96$, hence the ratio $\max (F(\theta)) / F(\underline{\theta}$ is about 9. Figure 8 below shows the simplest prediction scenario starting on Today $=T_{4}=$ $3-$ Jan -2021 . In the Legend of the Figure, Coef $1=1$ and Coef $2=1$ mean that no change by the authorities will be undertaken starting Today and ending on the Third restrictions date $=T_{5}=28-$ Jan -2021 . Further, Coef $11=1$ and Coef $22=1$ mean that no relaxation of the measures will follow starting on $28-$ Jan -2020 .

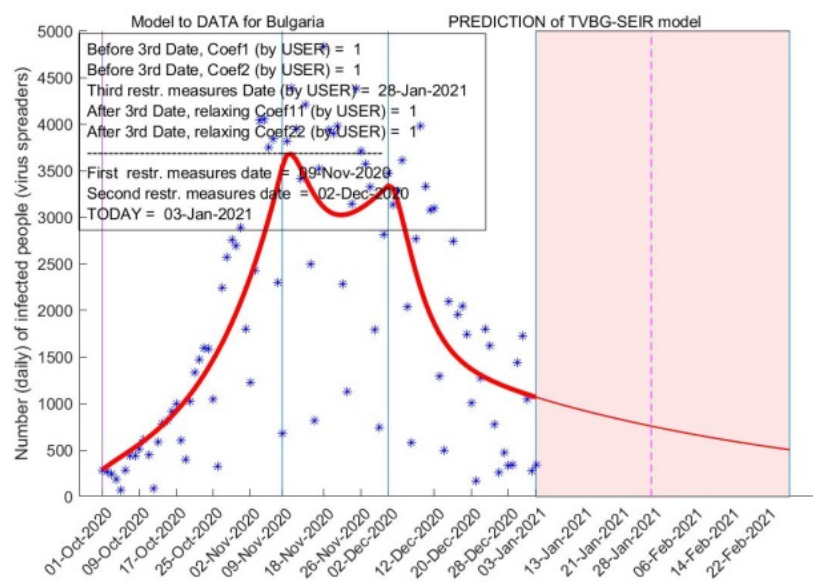

Figure 8.

4) However, on $28-$ Jan - 2021 only the measures decreasing the coefficient $\beta$ may be partially relaxed, without appearance of a "next wave", i.e. we may afford Coef $11=1.4$. This is seen on the Figure 9 below:

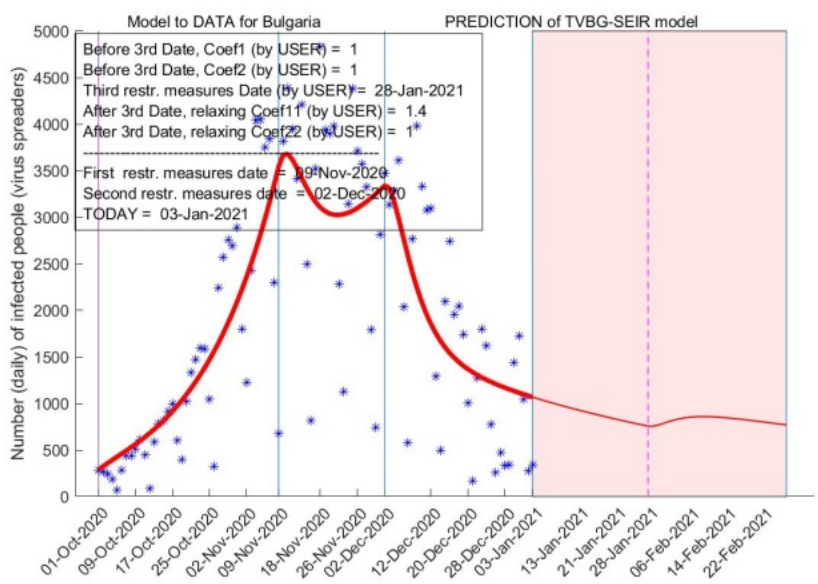

Figure 9.

From Figure 10 below we see that too much relaxing one of the measures, e.g. the first one, with Coef $11=1.8$, a "second wave" of infectious cases will arise in February 2021:

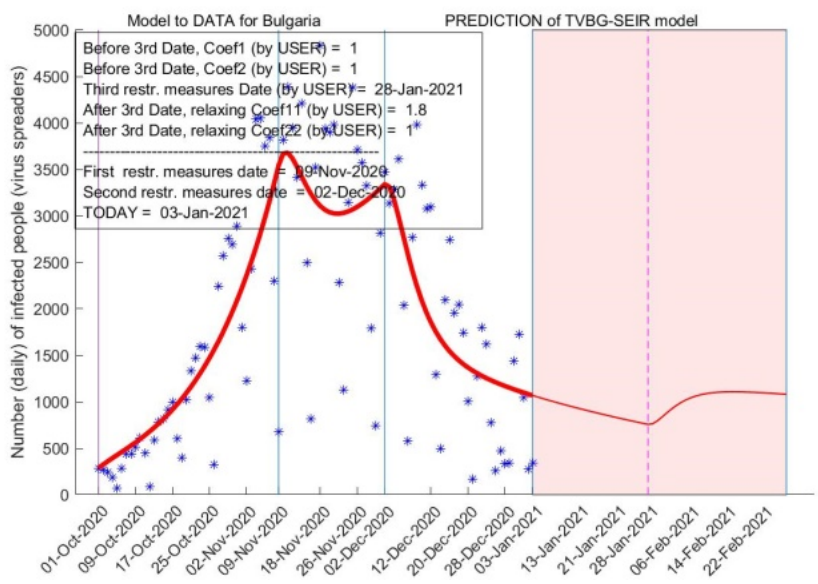

Figure 10.

The second wave is inevitable if more relaxation of the measures is allowed by the health authorities: namely, relaxing both measures, i.e. Coef $11=$ Coef $22=1.8$ after 28-Jan-2021 will generate a strong "next wave" of infections, as seen from Figure 11. 
O. Kounchev, G. Simeonov, Zh. Kuncheva, Scenarios for the spread of COVID-19 analyzed by ...

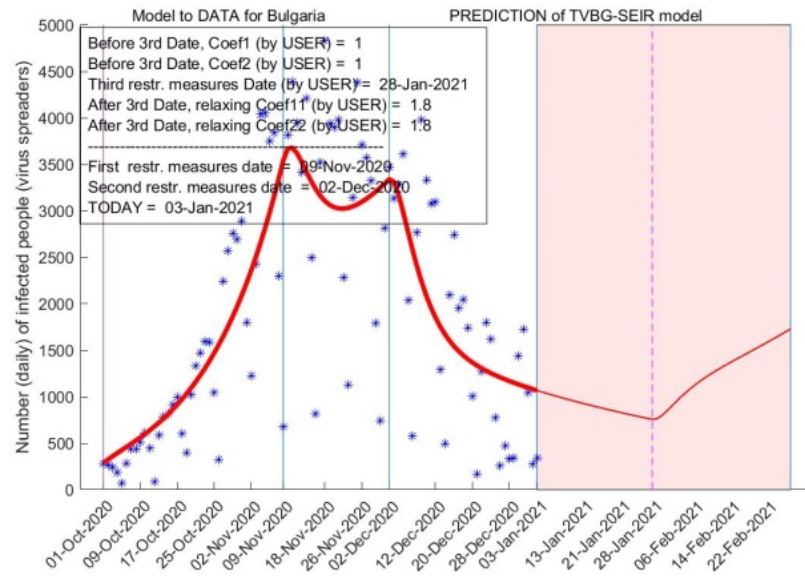

Figure 11.

5) Similar are the conclusions with Model3 for Bulgaria (with Fval $=31.60$, with "next wave" appearing as well.

6) For Model2 (with Fval $=31.78$ ) we have the most optimistic scenario since we may partially relax both measures after 28 - Jan - 2021 (i.e. Coef $11=$ Coef $22=1.4$ ), and no second wave will appear, as seen from Figure 12 below:

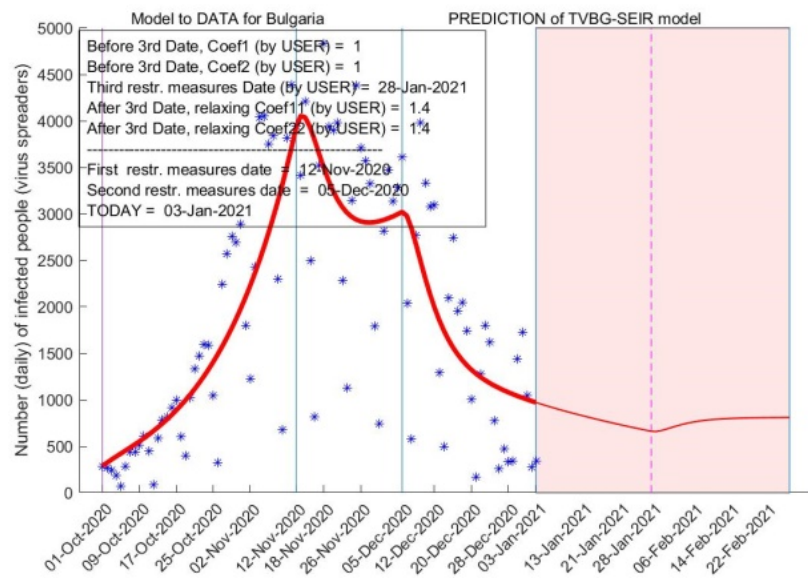

Figure 12.

As we mentioned above, we may use say ten models Model2, Model2,..., Model11 and generate their curves $I(t)$ to obtain estimate of the Confidence intervals at every time $t$. However we found the above presentation using "optimistic" and "pessimistic" scenarios more simple and clear.

\section{ApPlication to ITALian COVID-19 \\ DATA IN OCTOBER-DECEMBER, 2020, AND SCENARIOS GENERATED BY THE SBT-COVID19 TOOL}

In the present section we provide similar results obtained by our scenario SBT-COVID19 Tool for the Italian data.

1) The results about Italy considered till Today $=1-$ Jan -2021 are similar to Bulgarian. For Model 0 we have $T_{2}=6-N o v-2020, T_{3}=$ $20-N o v-2020$, and Fval $=F(\underline{\theta})=153.09$.

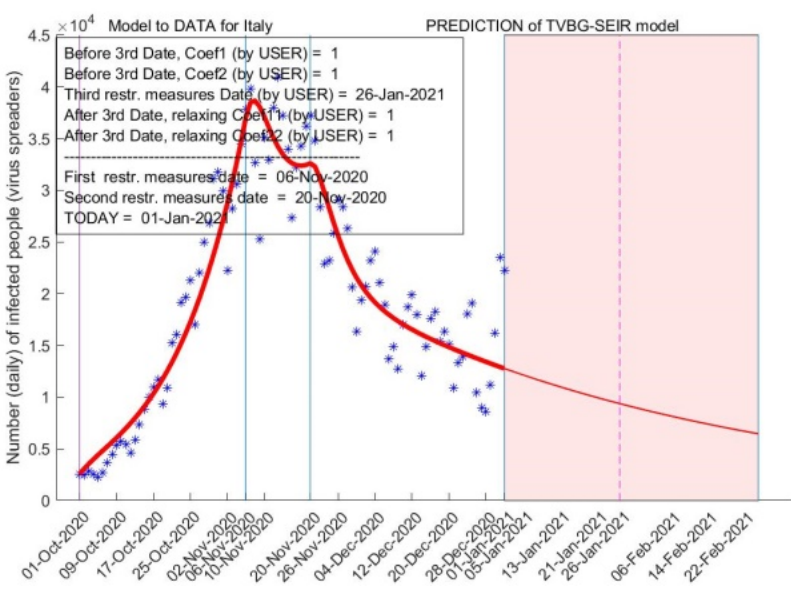

Figure 13.

A strong relaxation after the Horizon date 26Jan-2021 results in a strong "next wave" seen in Figure 14

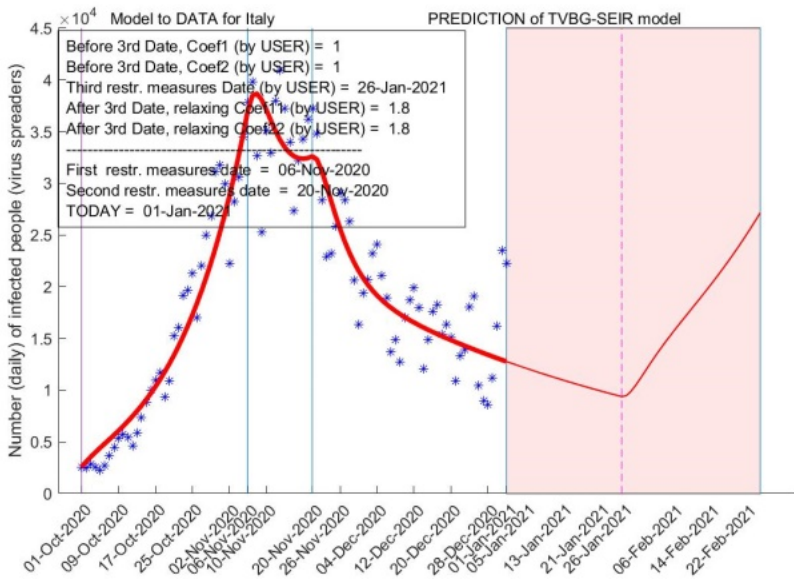

Figure 14. 
O. Kounchev, G. Simeonov, Zh. Kuncheva, Scenarios for the spread of COVID-19 analyzed by ...

2) Model 2 has $T_{2}=3-N o v-2020, T_{3}=$ $17-$ Nov -2020 , and Fval $=F(\underline{\theta})=159.77$. For it we obtain the following scenario:

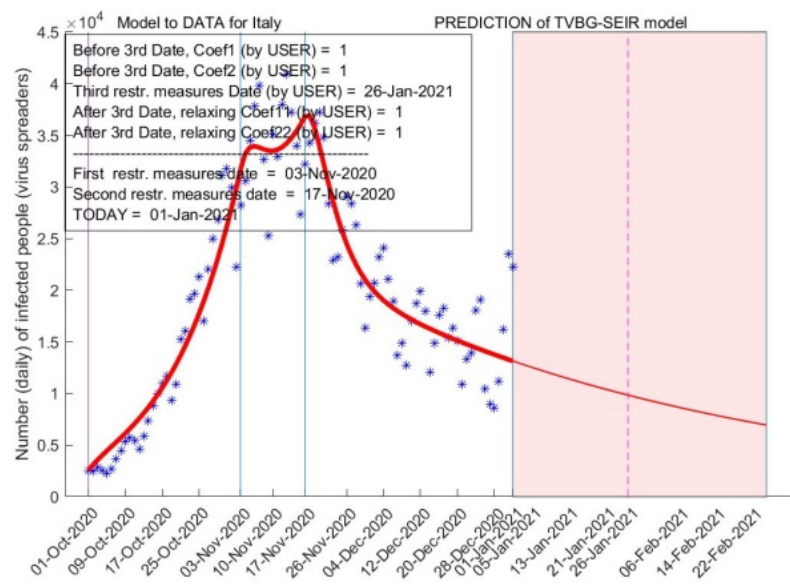

Figure 15.

Just as in Model1, further strong relaxation gives a strong "next wave" provided in Figure 16.

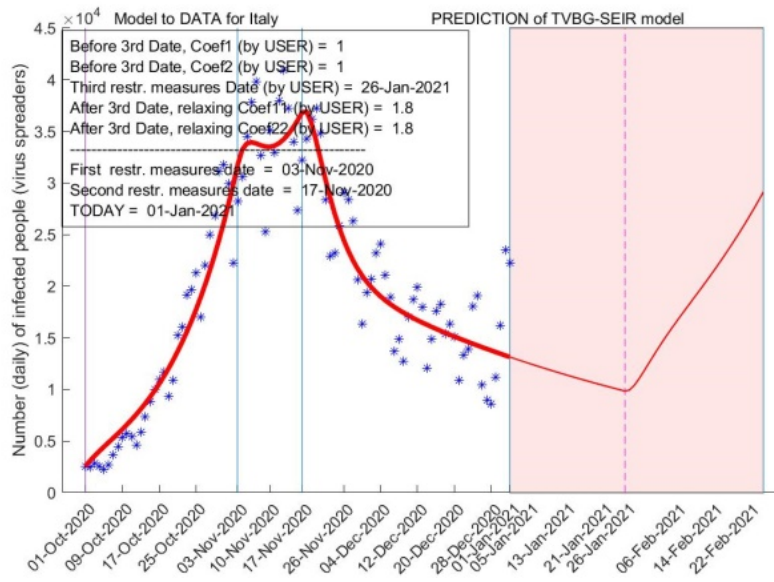

Figure 16.

Model 3 has $T_{2}=6-N o v-2020, T_{3}=23-$ Nov -2020 , and $F v a l=F(\underline{\theta})=169.72$. It gives the following Figure 17.

Further relaxation after $26-$ Jan -2021 shows a bigger "next wave" in the Figure 18.

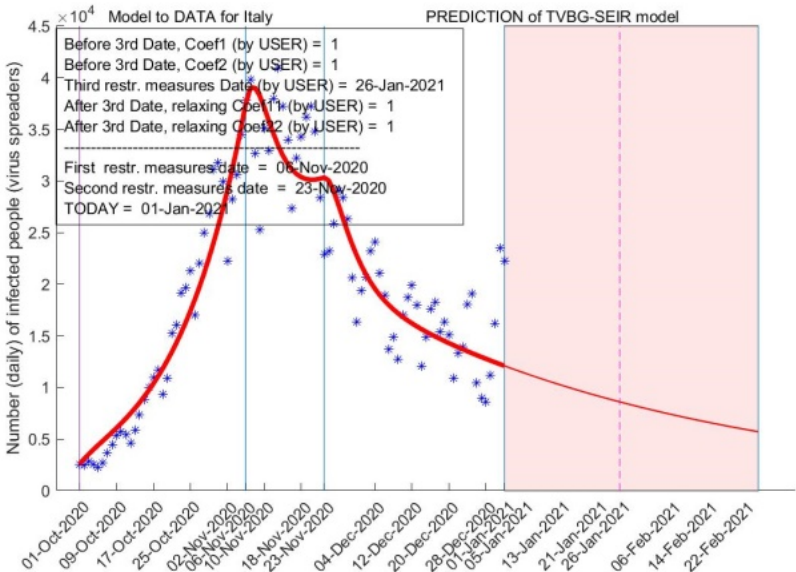

Figure 17.

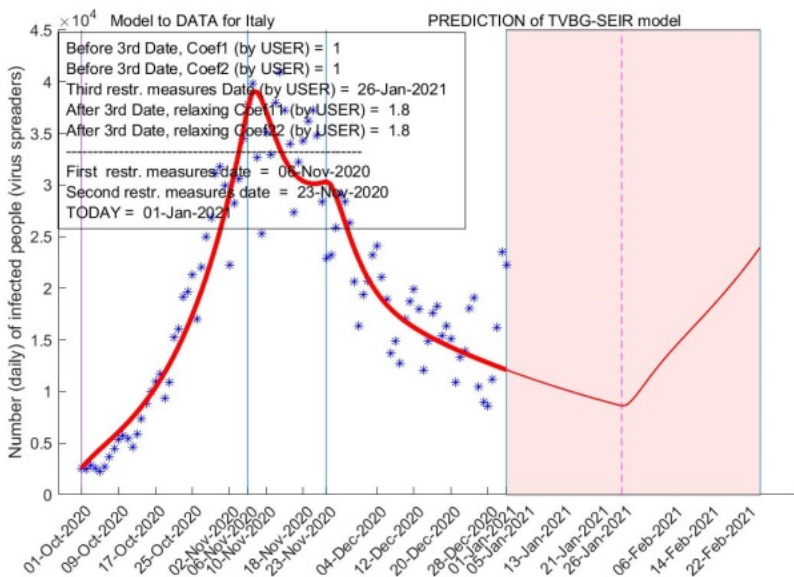

Figure 18

VII. APPLICATION OF THE MODEL TO GERMAN COVID-19 DATA IN MARCH-MAY, 2020, AND SCENARIOS GENERATED BY THE SBT-COVID19 TOOL

We provide the results for the German data. The overall feeling is, that unlike the data for Bulgaria and Italy, they show a very strong tendency to "explode" into a "next wave".

1) For the German data, on the Today date $=$ 1 - Jan - 2021, according to our Model1 for Germany, we have $T_{2}=3-N o v-2020, T_{3}=$ $11-$ Nov -2020 , and Fval $=F(\underline{\theta})=160.96$.

It is seen that if the containment measures remain the same as before Today, then Germany is already in the "next wave", which is seen from the Figure 19 below: 
O. Kounchev, G. Simeonov, Zh. Kuncheva, Scenarios for the spread of COVID-19 analyzed by ...

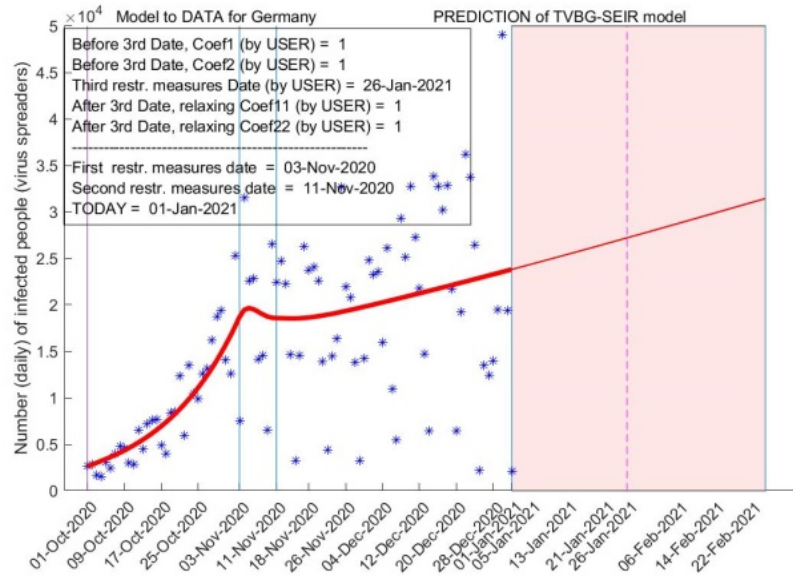

Figure 19.

2) However, a moderate tightening of the second measures $($ Coef $2=1.2)$ will result in a calming (Figure 20).

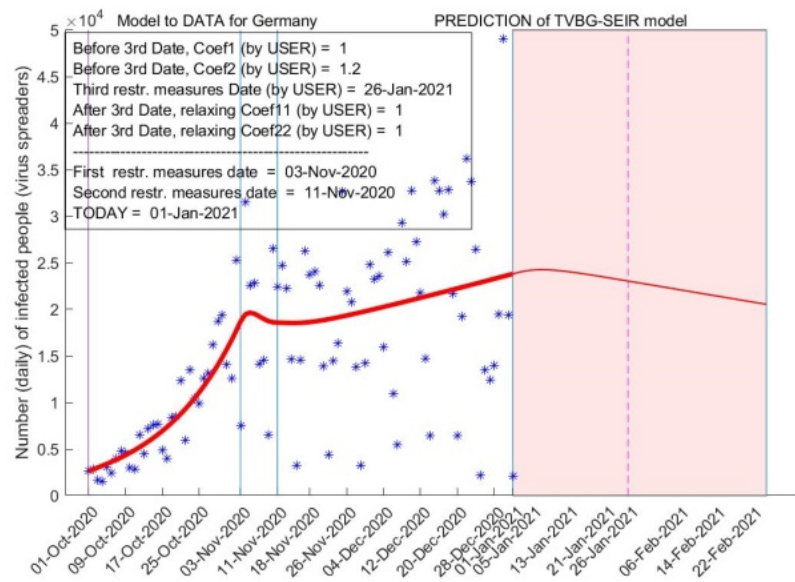

Figure 20.

But a much better result will bring the tightening of the measures influencing the coefficient $\beta$, i.e. Coef $1=1.2$ as seen in the Figure 21 .

3) For Model 2 we have $T_{2}=3-N o v-2020$, $T_{3}=8-N o v-2020$, and Fval $=F(\underline{\theta})=169.16$, and for Model $3-T_{2}=3-N o v-2020, T_{3}=$ $20-$ Nov -2020 , and Fval $=F(\underline{\theta})=171.61$. The application of the two models gives a result similar to that of Model1.

On the Figures 22 and 23 one may choose how strong the tightening of the measures has to be, within Model2, in order to obtain a stronger slowdown of the infection progression.

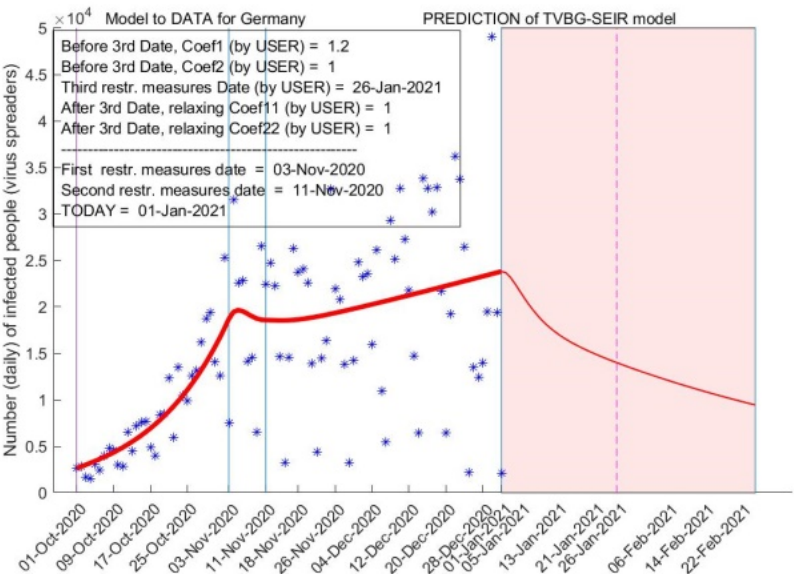

Figure 21

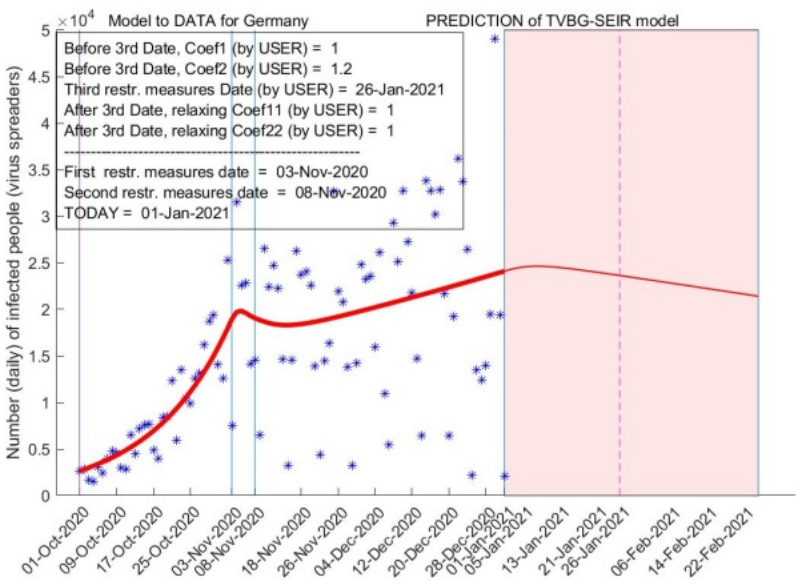

Figure 22.

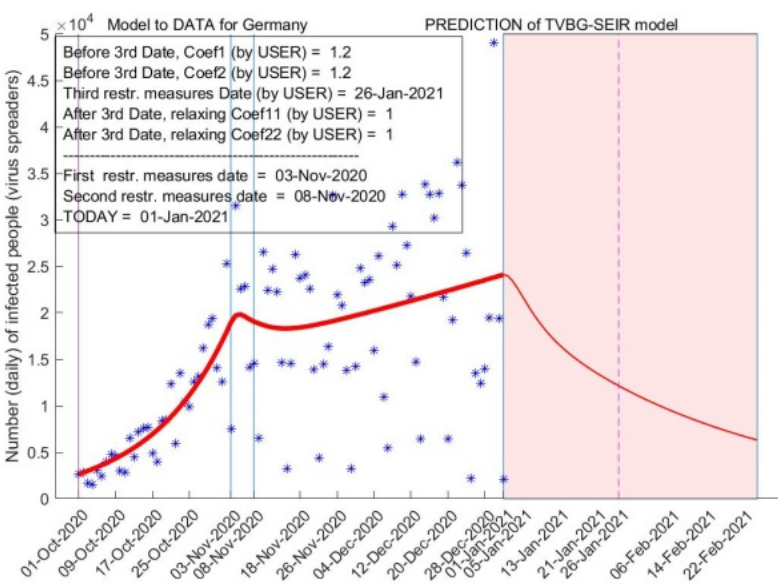

Figure 23. 
O. Kounchev, G. Simeonov, Zh. Kuncheva, Scenarios for the spread of COVID-19 analyzed by ...

And for Model3: we have a slight difference on Figure 24 below:

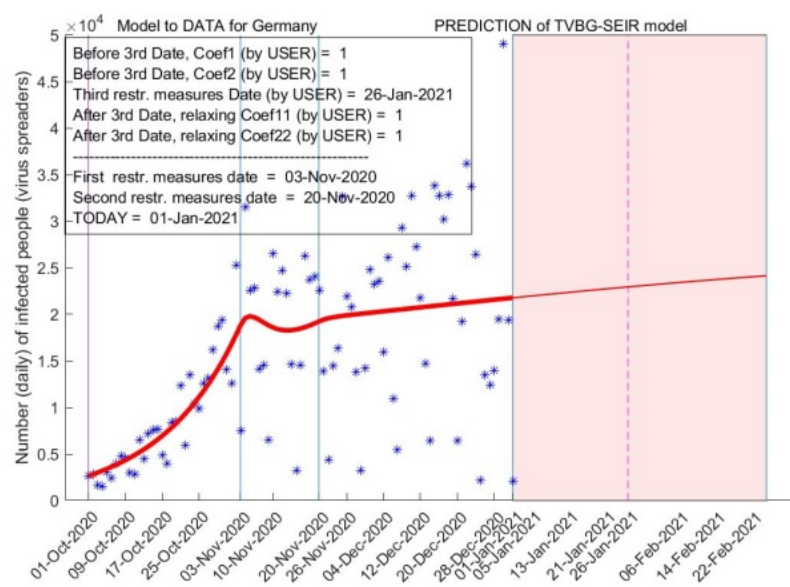

Figure 24.

\section{DESCRIPTION OF THE SBT-COVID19 TOOL FOR CONTROLLED SCENARIOS}

1) We have designed a SBT-COVID19 Tool for the Model predictions of the Corona virus (and similar infectious diseases) spread. The SBTCOVID19 Tool is based on the fitting of the TVBG-SEIR model to the official data available on a daily basis as described in Section II, B). The online tool is available at the site

http://213.191.194.141:8888/notebooks/

TVBG-SEIR-Spline-model_v3.ipynb?token=

b5d97bfbd7dd062e47ee7ab51837e470a8c226743a4667ee

2) First of all, we fit the model for the time series in the interval $\left[T_{1}, T_{4}\right]$, where $T_{4}$ is Today's date. Then the USER may choose several parameters to make a prediction about the virus spread during the period $\left[T_{4}\right.$, Horizon $]$, where Horizon is chosen to be at most 2 months from Today $=T_{4}$.

3) The first parameter, to be controlled, is the Third restrictive measures date denoted by $T_{5}$. The USER may choose several options, say 5, 15, 25 days from Today $\left(=T_{4}\right)$, i.e. one may select the dates $T_{5}=T_{4}+5, T_{4}+15, T_{4}+25$.

4) Then the USER may decide how to strengthen or relax the Beta measures and the Gamma measures during the period $\left[T_{4}, T_{5}\right]$, by means of the coefficients Coef 1 and Coef2, respectively; Coef $1=1$ means that the Beta measures remain the same, while Coef $2=1$ means that the Gamma measures remain the same in the period $\left[T_{4}, T_{5}\right]$. If Coef $1<1$, then this means that the Beta measures are "weaker", and also, the smaller Coef 1 the weaker are the Beta measures and they will reach a target value at the date $T_{5}$, which is defined by the size of Coef 1 (Note that Coef $1<1$ means that the rate $\beta(t)$ will be bigger!). In a similar way, if Coef $2<1$, then this means that the Gamma measures will be "weaker", and the smaller Coef 2 , the weaker are the Gamma measures (note that in such case the rate $\gamma(t)$ will be smaller!). A target value (determined by the size of Coef2) will be reached at the date $T_{5}$.

5) On the other hand, if Coef 1 or Coef 2 are bigger than 1, this means "strengthening the measures", resp. of Beta measures and Gamma measures in the period $\left[T_{4}, T_{5}\right]$ to some target value defined by Coef 1 , Coef 2 .

6) The USER has further the possibility to decide what will happen after date $T_{5}$ - to weaken or leave the same the Beta and the Gamma measures. This is decided by the choice of two coefficients Coef 11 for the Beta and Coef 22 for the Gamma measures. Coef $11=1$ means that one retains the same level of the Beta measures; Coef $22=1$ means that one retains the same level of the Gamma measures. If Coef $11>1$ then this would relax the Beta measures - the bigger Coef 11 the more the relaxation. Coef 22 makes the same for the Gamma measures.

7) A similar way to represent the above approach to Scenarios design and visualization is implemented since relatively recently in the popular online tool Covid-19 Projections, http://www. healthdata.org/covid/ (Initially, they used confidence intervals around the most probable scenario) Instead of using coefficients Coef 1 , Coef 2 , they use a more descriptive terminology for "worse" and "better" scenarios, as "95\% masks usage", "short lasting vaccination", etc. Their terminology changes very fast in time. 
O. Kounchev, G. Simeonov, Zh. Kuncheva, Scenarios for the spread of COVID-19 analyzed by ...

\section{RECENT RESEARCH ON TIME-VARYING TRANSMISSION RATES}

As we already said, presently it is urgent to consider SIR/SEIR models with time-varying $\beta(t)$ and $\gamma(t)$ rates.

Let us mention some research about solving an inverse problem for finding time-varying $\beta(t)$, in a SIR model, for a fixed removal rate $\gamma$, [10], from the number of infectious cases. In [1], the authors do research and provide further references of research on specific models for the transmission rate $\beta(t)$.

\section{ACKNOWLEDGements}

All authors acknowledge the support by grants KP-06-N32-8 and KP-06N42-2 of Bulgarian NSF. The first-named author acknowledges the partial support by Grant No BG05M2OP001-1.001-0003, financed by the Science and Education for Smart Growth Operational Program (2014-2020) and cofinanced by the European Union through the $\mathrm{Eu}-$ ropean structural and Investment funds.

All authors acknowledge the support of a fast-track grant by the European Open Science Cloud (EOSC) in the framework of the EOSCSecretariat.

The authors would like to thank the anonymous referee for the positive criticism which has essentially improved the paper.

\section{REFERENCES}

[1] Boatto, C. Bonnet, B. Cazelles and F. Mazenc, "SIR model with time dependent infectivity parameter: approximating the epidemic attractor and the importance of the initial phase", HAL, 2018, Id: hal-01677886, https://hal.inria.fr/hal-01677886

[2] Kermack, W. O. McKendrick, A. G., "A Contribution to the Mathematical Theory of Epidemics", Proceedings of the Royal Society A, 115 (772): 700-721, 1927.

[3] S. A. Lauer, K. H. Grantz, Qifang Bi, F. K. Jones, Qulu Zheng, H. R. Meredith, A. S. Azman, N. G. Reich, J. Lessler, (2020), "The Incubation Period of Coronavirus Disease 2019 (COVID-19) From Publicly Reported Confirmed Cases: Estimation and Application", Annals of Internal Medicine, Vol. 172, No. 9, May 2020, pp. 577-582, doi:10.7326/M20-0504.

[4] Keeling, M. J. and Rohani, P., (2008), "Modelling Infectious Diseases", Princeton University Press.
[5] E. Brooks-Pollock, G. O. Roberts and Matt J. Keeling, "A dynamic model of bovine tuberculosis spread and control in Great Britain", Nature 511 228-231, 2014, doi:10.1038/nature13529.

[6] Michael J. Tildesley, Thomas A. House, Mark C. Bruhn, Ross J. Curry, Maggie O’Neil, Justine L. E. Allpress, Gary Smith, and Matt J. Keeling, (2010), "Impact of spatial clustering on disease transmission and optimal control", Proc Natl Acad Sci USA 107 1041-6, doi:10.1073/pnas.0909047107

[7] M Woolhouse, M Chase-Topping, D Haydon, J Friar, L Matthews, G Hughes, D Shaw, J Wilesmith, A Donaldson, S Cornell, M Keeling and B Grenfell, (2001), "Foot-and-mouth disease under control in the UK", Nature 414, 258-258.

[8] A. J Kucharski, Timothy W. Russell, Charlie Diamond, Yang Liu, John Edmunds, Sebastian Funk, Rosalind M. Eggo, (2020), "Early dynamics of transmission and control of COVID-19: a mathematical modelling study", The Lancet, published online Published: 11 March 2020, doi:10.1016/S1473-3099(20)30144-4

[9] Marc Lipsitch, Ted Cohen, Ben Cooper, James M. Robins, Stefan Ma, Lyn James, Gowri Gopalakrishna, Suok Kai Chew, Chorh Chuan Tan, Matthew H. Samore, David Fisman, and Megan Murray, (2003), "Transmission Dynamics and Control of Severe Acute Respiratory Syndrome", Science, 20 June 2003, Vol. 300, pp. 19661970, doi:10.1126/science.1086616

[10] Mark Pollicott, Hao Wang, Howard Howie Weiss, (2012), "Extracting the time-dependent transmission rate from infection data via solution of an inverse ODE problem", 2012, doi:10.1080/17513758.2011.645510

[11] Stoer, J.; Bulirsch, R. (2002), "Introduction to Numerical Analysis", 3rd ed., Berlin, New York: SpringerVerlag.

[12] Biao Tang, Xia Wang, Qian Li, Nicola Luigi Bragazzi, Sanyi Tang, Yanni Xiao, Jianhong Wu, (2020), "Estimation of the Transmission Risk of the 2019-nCoV and Its Implication for Public Health Interventions", J. Clinical Medicine, 2020, 9, 462; doi:10.3390/jcm9020462

[13] Biao Tang, Nicola Luigi Bragazzi, Qian Li, Sanyi Tang, Yanni Xiao, Jianhong Wu, (2020), “An updated estimation of the risk of transmission of the novel coronavirus (2019-nCov)", Infectious Disease Modelling, Vol. 5, 2020, pp. 248-255, ISSN 2468-0427, doi:10.1016/j.idm.2020.02.001

[14] Wallinga, J. and M. Lipsitch, (2007), "How generation intervals shape the relationship between growth rates and reproductive numbers", Proceedings: Biological Sciences, Vol. 274, No. 1609, (Feb. 22, 2007), pp. 599604

[15] Jane M Heffernan, R. J. Smith and L.M. Wahl, (2005), "Perspectives on the basic reproductive ratio", J. R. Soc. Interface (2005) 2, Published online (7 June 2005), pp. 281-293, doi:10.1098/rsif.2005.0042

[16] T. Hastie, R. Tibshirani, J. Friedman, The Elements of Statistical Learning, Springer, 2009. 
O. Kounchev, G. Simeonov, Zh. Kuncheva, Scenarios for the spread of COVID-19 analyzed by ...

[17] O. Kounchev, G. Simeonov, Zh. Kuncheva, The TVBG-SEIR spline model for analysis of COVID-
19 spread, and a Tool for prediction scenarios, https://arxiv.org/abs/2004.11338 